\title{
Buscando renovar la praxis en la seguridad contemporánea: capacidades y formación por competencias
}

\author{
Looking to renew the praxis in contemporary security: capabilities and training \\ for competences
}

\section{Buscando renovar a práxis na segurança contemporânea: capacidades e formação por competências}

\author{
Alejandra Cerón Rincón ${ }^{\mathrm{a},}$ | David López Cortés \\ ${ }^{\text {a }}$ https://orcid.org/0000-0002-5642-7949 \\ ${ }^{b}$ https://orcid.org/0000-0002-3364-3825 \\ Escuela de Posgrados de la Fuerza Aérea, Bogotá, Colombia
}

- Fecha de recepción: 2019-07-21

- Fecha concepto de evaluación: 2019-09-16

- Fecha de aprobación: 2019-11-02 http://dx.doi.org/ | 0.22335/rlct.v I Ii3.978
Para citar este artículo / To reference this article / Para citar este artigo: Cerón Rincón, A., \& López, D. (2019). Buscando renovar la praxis en la seguridad contemporánea: capacidades y formación por competencias. Revista Logos Ciencia \& Tecnología, I I (3), 82-93. http://dx.doi.org/ I0.22335/rlct.vI I i3.978

\begin{abstract}
RESUMEN
Después de la revisión teórica, conceptual y metodológica sobre los abordajes académicos y prácticos a la seguridad, hoy se plantea como ejercicio reflexivo la pertinencia de los contenidos curriculares y de los métodos de enseñanza dentro de la Maestría en Dirección y Gestión de la Seguridad Integral (MADGSI) de la Escuela de Posgrados de la Fuerza Aérea Colombiana. Mediante el concepto de capacidades acuñado por Amartya Sen y del enfoque educativo por componentes, se desarrolla una discusión diagnóstica acerca de las oportunidades de mejora para el programa académico para ampliar los conocimientos teórico-prácticos de los estudiantes de acuerdo con sus experiencias profesionales y las demandas de las dinámicas contemporáneas de la seguridad. A partir de un ejercicio de consulta a los miembros de la comunidad educativa involucrada, a través de encuestas y entrevistas, se conjuga la reflexión teórica en torno a la seguridad con sus percepciones actuales y expectativas acerca del trabajo. Se concluye con una discusión abierta sobre la necesidad de ampliar el componente investigativo para incrementar las capacidades de acción de los estudiantes de la MADGSI.
\end{abstract}

Palabras clave: seguridad, enseñanza, competencias para la vida, seguridad del Estado, seguridad humana 


\section{SUMMARY}

After the theoretical, conceptual and methodological review of the academic and practical approaches to security, the relevance of curricular contents and teaching methods is considered to be a reflective exercise within the Master's Degree in Direction and Management of Integral Security at the Colombian Air Force's Postgraduate School. Through the concept of skills coined by Amartya Sen and the educational approach by components, a diagnostic discussion is developed related to the improvement opportunities for the academic program to expand the theoretical and practical knowledge of the students according to their professional experiences and demands of contemporary security dynamics. From a consultation exercise to the members of the educational community involved, through surveys and interviews, the theoretical reflection on safety is combined with their current perceptions and expectations regarding work. It concludes with an open discussion about the need to expand the research component in order to increase the action capabilities of the students.

Keywords: security, education, life skills, state security, human security

\section{SUMÁRIO}

Após a revisão teórica, conceitual e metodológica das abordagens acadêmicas e práticas à segurança, hoje se busca como exercício reflexivo a pertinência dos conteúdos curriculares e dos métodos de ensino dentro do Mestrado em Direção e Gestão da Segurança Integral (MADGSI) da Escola de Pósgraduações da Força Aérea Colômbiana. Mediante o conceito de capacidades cunhado por Amartya Sen e do enfoque educativo por componentes, desenvolve-se uma discussão diagnóstica sobre as oportunidades de melhora para o programa acadêmico para ampliar os conhecimentos teóricopráticos dos estudantes de acordo com suas experiências profissionais e as demandas das dinâmicas contemporâneas da segurança. A partir de um exercício de consulta aos membros da comunidade educativa envolvida, através de pesquisas e entrevistas, se conjuga a reflexão teórica em torno da segurança com suas percepções atuais e expectativas sobre o trabalho. Conclui-se com uma discussão aberta sobre a necessidade de ampliar o componente investigativo para incrementar as capacidades de ação dos estudantes da MADGSI.

Palavras-chave: segurança, ensino, competências para a vida, segurança do Estado, segurança humana

Este escrito es resultado de un proceso investigativo en el que se pretendió evaluar el valor de la seguridad dentro del programa de Maestría en Dirección y Gestión de la Seguridad Integral (MADGSI) de la Escuela de Posgrados de la Fuerza Aérea Colombiana (EPFAC). La profundización de las discusiones académicas que suscitan los nuevos desafíos de la seguridad conlleva la apertura de otras maneras de desarrollar los currículos académicos y las prácticas docentes e investigativas. Esto requiere agudizar los fundamentos metodológicos y conceptuales, volviendo a las humanidades, como la filosofía o la psicología, $y$ a las ciencias sociales, como la sociología o la economía.

Durante la reconceptualización de la seguridad, se traen a la discusión académica los cambios en las relaciones y los fenómenos de la modernidad a la época actual. Entre estos se encuentra la globalización, una temática amplia y compleja que afecta en su esencia a la definición de seguridad, sus actores y canales de interacción. ¿Cómo se conectan los avances teóricos con las prácticas de los actores involucrados en el rubro de la seguridad?

La relación disciplinar inmediata de los estudios en seguridad con la ciencia política y las relaciones internacionales arrastra avasalladoramente hacia la profundización de las investigaciones y la reproducción de las cátedras universitarias dentro de los límites del Estado y del poder político/militar. No obstante, al incluir los factores de cambio que requiere la época, los conceptos de seguridad mutan y se amplifican sus alcances;se empieza a escribir sobre la seguridad multidimensional o sobre la seguridad integral, al mismo tiempo que las acciones y planes de los gobiernos asignan nuevas funciones a las instituciones militares y policivas. La MADGSI pertenece a esa renova- 
ción académica en torno a las reconceptualizaciones de la seguridad. Ahora, ¿cuál es el alcance de esta propuesta posgradual en las prácticas y experiencias de sus estudiantes? ¿Qué debe cambiar y qué es inamovible?

Esta investigación tuvo como centro de interés conocer los aportes de la MADGSI a las labores investigativas de sus estudiantes, en tanto los provea de capacidades y de competencias para la realización de sus labores como profesionales en seguridad integral, sea desde el ámbito militar, gubernamental o estrictamente académico. En consecuencia, este texto se plantea más allá de un informe de investigación, como una propuesta de renovación curricular y pedagógica del programa debido al reconocimiento de la importancia del proyecto que representa la MADGSI en las discusiones académicas sobre la seguridad en Colombia.

El artículo desarrolla dos temáticas principales: la primera, la presentación de los resultados de la investigación realizada al interior de la MADGSI, evidenciando la metodología de intervención y su relación con el propósito misional de la consolidación y el lanzamiento del programa académico; en la segunda, se justifica el avance de la investigación con el discernimiento teórico sobre las capacidades de Sen (1999) con el direccionamiento del currículo y de la pedagogía de la maestría hacia un enfoque de competencias.

\section{Nuevos debates y conceptos en torno a la seguridad}

La academia y la política han sido reactivas para los fenómenos y desafíos que postulan las realidades nacionales y globales que se transforman y afectan las condiciones de vida y las interacciones entre seres humanos. Pese a que las consideraciones sobre la seguridad diferentes de las amenazas militares de otros estados siempre existieron y pudieron haber sido de interés del Estado, ha sido reciente la atención prestada, desde la seguridad, a otras temáticas. Esto implica nuevas funciones, relaciones y aprendizajes de las agencias públicas y privadas de seguridad; al mismo tiempo, se demandan nuevas concepciones que permitan tales relacionamientos específicos con los cambios de panorama.

En las teorías clásicas de las relaciones internacionales, la seguridad era un concepto estado-céntrico: la configuración de un sistema internacional en tensión constante de potenciales conflictos con otros Estados.
En especial, el realismo propendió a explicar la seguridad como el leitmotiv de la actividad estatal, delegado especialmente al poderío militar. De esta forma, la razón del Estado era el aseguramiento de la supervivencia a través de una seguridad militarizada. De aquí se destaca una concepción de seguridad física, de la protección de los recursos naturales y de las condiciones materiales de la población (Morgenthau, 1948).

En esta concepción realista de la política, el papel de las fuerzas militares, e incluso de la policía, es la manutención del orden desde la disuasión o de la acción militar. Es más, la estrategia militar se concentró exclusivamente en operaciones de ataque o de defensa combinando las técnicas y especialidades de cada fuerza. Incluso, el desarrollo de la industria militar fue fundamental para obtener ventaja tecnológica sobre cualquier posible adversario. En síntesis, la producción de la doctrina de seguridad estuvo íntimamente ligada a las militares y policivas, como fuerzas preparadas para cualquier eventualidad conflictiva.

Especialmente, al final de la Guerra Fría empiezan a ser evidentes ciertos cambios en la modernidad, sobre todo por sus implicaciones globales. A este conjunto de regularidades se le llamó "globalización"; los espacios transnacionales emergen de diversas formas, gracias a que nuevos canales de comunicación se abren para diversas sociedades y contactos. Se volvió casi innegable el tránsito hacia la globalización: mercado internacional, migraciones, sociedades globales, intercambios culturales, tecnologías de la información, etc.

Estas incesantes interacciones transnacionales acapararon la atención de la academia, al dudar de la existencia de un orden mundial establecido. Si bien el concepto 'globalización' no es preciso para la explicación de los "espacios sociales transnacionales" (Beck, 2008), su uso extensivo en la política y en los medios de comunicación ha abierto el debate de las nuevas funciones del Estado, de la sociedad civil y de los actores privados en las crecientes relaciones transnacionales. Los ejercicios de reflexión de los académicos de las ciencias sociales han llevado al planteamiento de diversas explicaciones de los recientes acontecimientos transnacionales; mientras unos apoyan la tesis de una creciente 'globalización', otros niegan su novedad.

En el ámbito de la seguridad también surgieron afectaciones importantes a partir de nuevas experiencias internacionales y de otros conceptos añadidos a sus consideraciones académicas y políticas. En efecto, la definición de seguridad aparece como "contingentemente contestada", ajustada a 
la experiencia y reflexión política de los actores (Booth, 2007). En este punto, es plausible afirmar que existe una variedad de conceptualizaciones válidas de la seguridad, estrictamente desde la academia o desde la política. Sin embargo, existen elementos inherentes del concepto que no escapan en la elaboración contingente de los actores: en primer lugar, se entiende que la inseguridad es una condición para la supervivencia y la vivencia (Booth, 2007); en segundo lugar, la seguridad puede tener un valor instrumental cuando hay supervivencia asegurada y miedo a las amenazas, dando lugar para el establecimiento de condiciones de vida específicos (Booth, 2007); en tercer lugar, la seguridad tiene un potencial discursivo en la política debido al poder simbólico que contiene y que puede transmitir a diversos públicos para definir agendas políticas (Booth, 2007); en cuarto lugar, la seguridad es un concepto que puede derivar de los lineamientos y propósitos políticos generales (Booth, 2007). En última instancia, la seguridad tiene como precondición a la supervivencia, pero debe indicarse de manera tautológica como fin en sí misma porque tiene lugar en el sentir la libertad de ser $y$ hacer.

Estas reflexiones parten de un cambio en la concepción epistemológica que guía la definición de la seguridad. Al menos en la teoría de las relaciones internacionales, la introducción constructivista a la seguridad hecha por Barry Buzan advirtió que la fuente de la seguridad yace en la sociedad, en su propia identificación de las amenazas y riesgos. Sucede, entonces, el paso de una concepción tradicional de la seguridad, como defensa de la soberanía y del territorio, hacia la "seguridad societal" en atención a la cultura e identidad de una sociedad. Por ejemplo, como la noción de seguridad depende de la identidad y las creencias de la sociedad, entonces con la "securitización" se da cuenta de cómo una temática puede convertirse en un problema de seguridad para esa sociedad (Thelier, 2010). Esta definición del problema es resultado de una controversia ocasionada por un evento interno o externo en relación con los valores y las motivaciones sociales. Por lo cual, las amenazas también se trasladan al interior del Estado y de las interacciones sociales.

Con este "giro epistemológico", la seguridad se convierte en un tema más maleable e inestable, dado que ahora aprehende una complejidad improvista, por demás incompatible con la formación educativa y académica de las agencias de seguridad estatales e internacionales. En parte, este debate se ha trasladado hacia el terreno de lo lingüístico para la comprensión de los movimientos narrativos y comunicativos en la opinión pública. Por demás, con la "securitización" debe percibir las actitudes de amenaza, las posturas defensivas y las afiliaciones ideológicas para suscribir los clamores de los grupos en torno a la seguridad dentro de una movilización específica (Thelier, 2010).

Otros avances teóricos sucedieron al conectar los estudios en seguridad con el desarrollo humano cuando se concentran los esfuerzos en el bienestar individual y social. Este enfoque tiene origen en el reporte del Programa de Naciones Unidas para el Desarrollo (PNUD), publicado en 1994, en el cual se propone un acercamiento centrado en la persona. En especial, este enfoque busca la libertad para que los individuos puedan realizarse sin miedos ni impedimentos (Peoples \& Vaughan-Williams, 20I0). Esta afirmación resulta bastante complicada para la aplicación de la seguridad humana, ya que según esta definición el individuo debería vivir en las mejores condiciones posibles para tener una vida digna y autónoma: protegido de la violencia, de la pobreza, de las necesidades básicas insatisfechas, de las enfermedades y adicciones, etc.

Por un lado, el Estado puede combatir el crimen internacional y el tráfico de personas para disminuir los riesgos a modo de prevención de la inestabilidad social; por otro, el Estado debe procurar disminuir la desigualdad económica y asegurar la educación y un clima económico favorable, lo que implica cambios en la gestión pública, como lo menciona Aparicio (2019): "La seguridad ciudadana representa un viraje en el gasto público e implica la asignación de más recursos a las organizaciones públicas que enfrentan el delito o garantizan la convivencia" (p. 25). La idea general es que, si aumenta el desarrollo y el bienestar, las personas van a sentirse más seguras para vivir con libertad, puesto que se prevendrían las amenazas físicas y las perturbaciones a la vida digna. Consecuentemente, las acciones del Estado y de las instituciones internacionales deben estar encaminadas para alcanzar el desarrollo humano. Esto implica acciones transformativas dentro de la composición de las instituciones del Estado, especialmente en las funciones y capacidades de las agencias de seguridad y de planeación'.

En el 2002, la extensión política internacional de los nuevos debates sobre la seguridad llegó a la asamblea de Bridgetown de la Organización de Estados Americanos (OEA), momento en el que se abordó la necesaria renovación

I Para profundizar en la transformación de los roles de las fuerzas policiales en la reconceptualización de la seguridad contemporánea, véase Aparicio (2019). 
conceptual de la seguridad. Allí, y con la Declaración sobre la Seguridad de las Américas de 2003 en Ciudad de México, se consagró el concepto de "seguridad multidimensional": una aspiración de homogeneizar la agenda de seguridad del continente en respeto de los valores democráticos, los derechos humanos, la paz, la solidaridad y la soberanía nacional. En la Declaración del 2003, los Estados americanos reconocen los nuevos desafíos a la seguridad, especialmente tratados por la seguridad humana, fomentando la prevención y la gestión efectiva de amenazas tradicionales y no tradicionales (OEA, 2003).

En esa necesidad, y posterior reconocimiento de los cambios conceptuales y fácticos de los temas de seguridad, el Estado y la academia han procurado realizar transformaciones institucionales y de política pública para cambiar los procedimientos y acciones de manera consecuente con estos cambios. Dentro de estos se encuentra la preocupación por afectar las prácticas mediante la educación de los agentes de seguridad del Estado y de los investigadores.

En concordancia con estos compromisos políticos y académicos, para el 2016, la Escuela de Posgrados de la Fuerza Aérea Colombiana (EPFAC) abrió el programa de Maestría en Dirección y Gestión de la Seguridad Integral (MADGSI) con la finalidad de

promover la formación de un profesional ético, crítico, comprometido, participativo con visión, capaz de afrontar creativamente los desafíos de seguridad integral, dirigir y gestionar procesos de seguridad integral, desarrollar proyectos en el campo de la seguridad integral y adelantar procesos de investigación en ambientes de la seguridad integral que aporten a la construcción de conocimiento de utilidad organizacional y social a nivel institucional, nacional, regional e internacional (EPFAC, 20I5, p. 36).

Siendo un programa de posgrado de una academia de las fuerzas militares, la MADGSI busca acoplarse con los fundamentos de la política pública en seguridad y con los compromisos internacionales adquiridos por Colombia; i. e., la "seguridad multidimensional" de las declaraciones de Bridgetown y Ciudad de México.

La MADGSI tiene como objetivo formar profesionales para que puedan ocupar cargos directivos, gerenciales e investigativos en relación con la seguridad integral en instituciones públicas o privadas; los profesionales podrán fomentar procesos de protección de infraestructura $y$ de activos vitales para los intereses del país mediante el fomento de una cultura organizacional que permita la reacción frente a eventos específicos (EPFAC, 20I5). Este modelo proviene del proyecto pedagógico de la EPFAC que prioriza una educación integral y flexible, ajustada a la construcción del conocimiento desde la relación propia estudiante-docente, lo cual permite motivar a la investigación autónoma, reflexiva e interdisciplinar. En especial, desde este modelo constructivista de la educación se propende por una destacada integración entre el conocimiento teórico y la praxis militar, policial o gerencial en la resolución de problemas y en la construcción de proyectos (EPFAC, 20I5).

Al observar la constitución del programa y del currículo académico, es inmediato el reflejo de la intención por formar profesionales especializados en gerencia y dirección de acciones compuestas e integrales para las amenazas multidimensionales que enfrentan las fuerzas militares y policiales, los gestores de política pública, la sociedad civil, el sector privado y la academia. Este enfoque en la planeación estratégica lleva a la organización efectiva de los recursos para la innovación de respuestas a dichos desafíos a la seguridad, expresado en nueve competencias propuestas por la MADGSI: formulación estratégica, compromiso fundamental para el cumplimiento de objetivos, investigación y generación de conocimiento nuevo, comportamiento ético, comunicación efectiva, responsabilidad social, resolución de problemas, toma de decisiones y sinergia organizacional. Sin embargo, cabe preguntarse: ¿es suficiente este acercamiento gerencial a los fenómenos y debates teóricos contemporáneos sobre la seguridad?

\section{Metodología de investigación diagnóstica para} la renovación curricular

El diseño metodológico para esta investigación combinó un enfoque mixto con técnicas cualitativas y cuantitativas. Con el consentimiento y la participación de los docentes, directivos e investigadores de la maestría de la EPFAC y expertos en seguridad ${ }^{2}$, se recogió una serie de entrevistas semiestructuradas hechas a los docentes y expertos, durante el primer semestre de 2018, alcanzando 14 en total, con el objetivo de encontrar en el discurso las narrativas, expectativas, críticas y apoyos al camino emprendido

\footnotetext{
2 La investigación se desarrolló en colaboración con la Comunidad Latinoamericana de Consultores y Asesores en Gestión de Riesgos y Seguridad (Coladca), que permitió el contacto y entrevista personal con algunos de sus miembros fundadores.
} 
después de la apertura del programa académico. De igual manera, y durante el mismo período, se aplicó un formato de encuesta a una muestra representativa de la comunidad académica, estudiantes y profesores, donde se les preguntaba, a modo de ejercicio reflexivo y crítico, sobre los componentes y las capacidades fomentadas y demandadas por el aprendizaje, y por la relación del conocimiento adquirido con las prácticas y funciones profesionales. Para la aplicación del instrumento se realizó una prueba piloto con una muestra de 5 encuestas, y posteriormente se aplicó a 27 participantes: 10 profesores y 17 estudiantes del programa MADGSI, desarrollando un total de 20 preguntas.

También, como paso preliminar a la aplicación de los métodos descritos, se requirió de una observación y análisis del contenido del currículo y metodologías pedagógicas empleadas en la MADGSI, momento para refutar la coherencia y la integralidad de los seminarios, la pertinencia de las teorías abordadas y la gradualidad del impacto de los métodos de enseñanza con respecto a los objetivos prácticos de la maestría. En este ejercicio fue necesario hacer un estado del arte alrededor de la seguridad para contrastar las temáticas y las teorías aplicadas en la maestría frente a otras alternativas de estudio y de investigación, que incluso podrían ser más adecuadas para tratar los debates más contemporáneos de la seguridad.

Por una parte, el método cualitativo de la entrevista semiestructurada permitió una conversación guiada entre el entrevistador y el entrevistado que dio la posibilidad de interactuar con las narrativas y las emociones, mientras se llevó el hilo conductor deseado de las temáticas a abordar durante la conversación. Afín a esta metodología se utilizó una técnica hermenéutica para conectar los testimonios con los referentes simbólicos; dado que la interpretación correcta de la postura que adquiere el otro frente a un estímulo específico durante la interacción, permite mantener la fluidez $y$, en parte, la autenticidad de la información proveída por el entrevistado (Denzin, 1978). La entrevista semiestructurada da la posibilidad al entrevistador para que tome caminos espontáneos en correspondencia con el sentido que adquiere la conversación, sea para adquirir información detallada o para refinar algún concepto valioso para la investigación. Además, fue valioso contar con la especificidad de cada sujeto entrevistado, en su experiencia como militar, policía, funcionario público o académico, para complementar las dimensiones de la apreciación general del Estado y del funcionamiento de la MADGSI.
Además, el método cuantitativo de la encuesta es un sondeo de una muestra de la población o en contados casos, si es posible, de su totalidad. El uso de la encuesta radica en la medición estimada del grupo objetivo del estado de opinión, del conocimiento y de las relaciones, mediante una selección representativa. Dependiendo de la aplicación de la encuesta va a variar la confiabilidad de los datos obtenidos, pero dado que esta se realizó de manera presencial, su aplicación resultó ser más efectiva y la indagación más directa y precisa. No obstante, es inevitable el sesgo de las preguntas con opción múltiple cuando las preguntas y las respuestas delimitan fuertemente los datos; conociendo el mantenimiento del sesgo durante todo el proceso de recolección de información, debe optarse por seleccionar la parcialidad de las respuestas para obtener información más afinada (Johnston, 2008). Estimando las posibles reacciones y las conocidas, se debe propender por confirmar o refutar las hipótesis, y por resolver las incógnitas de las observaciones previas. Por supuesto, esto depende de la claridad de los términos $y$ de que sean mutualmente excluyentes.

A partir de la aplicación de la encuesta a los estudiantes de la MADGSI, se buscaron las percepciones acerca de los aportes teórico-prácticos de los contenidos y desarrollos pedagógicos de las clases a sus propias experiencias como profesionales de la seguridad, en cualquier instancia.Además, se buscó el reconocimiento de las falencias y debilidades de los estudiantes, las cuales podrían superarse a través del programa académico; en este instrumento se incluyeron preguntas que profundizaron en el carácter investigativo de la MADGSI.

El instrumento constituido para la encuesta está compuesto de dos partes: preguntas abiertas y preguntas cerradas. Para las primeras se dejó el espacio para la expresión sucinta de los encuestados y luego se agruparon las respuestas en categorías generales; para las segundas, se utilizó la modalidad de respuestas medidas por escala de relevancia. Como resultado del ejercicio, se elaboraron algunas estadísticas relevantes en tablas y gráficas, presentadas a continuación (véanse figuras I y 2 ).

En la figura I se muestra el sondeo de las habilidades que los estudiantes de la MADGSI deben desarrollar, las cuales son consideradas como necesarias para ser proficiente en la dirección y en la gestión de temas relacionados con la seguridad. Se observan cuatro respuestas que están incluidas dentro de un método de investigación: abstracción 
A2. Habilidades que deben desarrollar los estudiantes de la MAGDSI

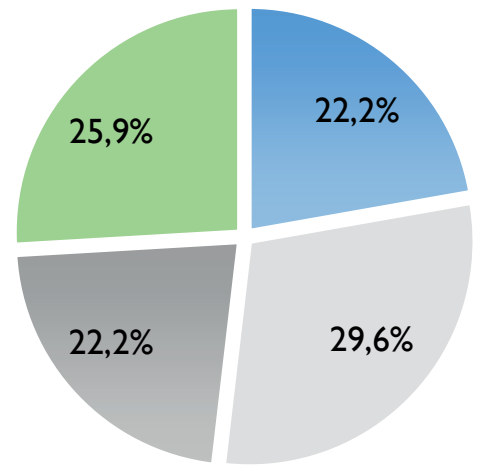

- Abstracción de información concreta y actual

- Investigación en gestión de riesgos y contexto se seguridad

- Análisis de pensamiento crítico y metodológico

- Conceptualización y lectoescritura

Figura I. Percepciones sobre las habilidades que necesita el profesional en seguridad integral

de la información observada, análisis crítico de la información, conceptualización acorde con los riesgos y los contextos de seguridad. En especial, esta figura ilustra la importancia de la formación como investigador para manejar los temas actuales de la seguridad. En complemento con lo extraído de la primera, las figuras subsiguientes van a destacar la importancia de la investigación en la construcción del profesional en sus diversas aplicaciones prácticas dentro de los ámbitos de la seguridad.

La figura 2 destaca, en la pregunta A4.I, que las competencias investigativas tienen relevancia para el $96,3 \%$ de la población encuestada, específicamente lo relativo a la identificación de problemas $(25,9 \%)$, el reconocimiento del contexto laboral $(44,4 \%)$ y la capacidad de reacción para tomar decisiones $(25,9 \%)$. En especial, esta relevancia es de tipo práctico, un reconocimiento importante de la relación teoría-praxis, en tareas fundamentales de autorreferenciación y respuesta programada a estímulos específicos por la incorporación cognitiva de conocimientos y habilidades nuevas.

Las figuras apuntan a las capacidades y competencias obligatorias para cualquier investigador. En primera instancia, se refleja la búsqueda de los estudiantes en el programa académico por mejorar sus métodos de investigación, al igual que afinar la observación y las habilidades de interpretar y reportar datos. En segunda, se muestra la relación entre las metodologías utilizadas en clase con las competencias investigativas para el campo laboral. La distribución porcentual de las respuestas se produjo con relativa equidad, pero reafirma el valor de las actividades académicas para la práctica laboral: planteamiento de argumentos académicos, fomento al liderazgo, aprendizaje general de conceptos y posibilidad creativa de explorar temáticas y métodos de investigación y análisis.

Si bien la reconceptualización de la seguridad surge como una respuesta teórica a las emergentes dinámicas de las coyunturas mundiales después del fin de la Guerra Fría, las reformas pedagógicas deben comprender las transformaciones estructurales de las funciones y capacidades de las instituciones militares y policiales. De la misma manera que la seguridad nacional transita hacia

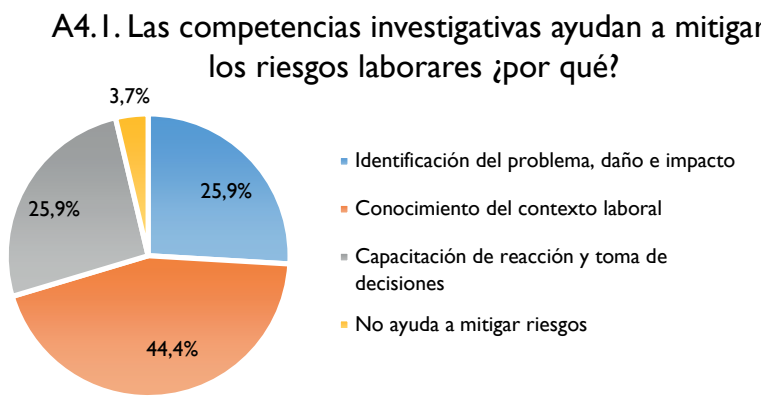

A6. Cómo evidencia en el aula la apropiación y aplicación de las competencias investigativas encaminadas al campo laboral

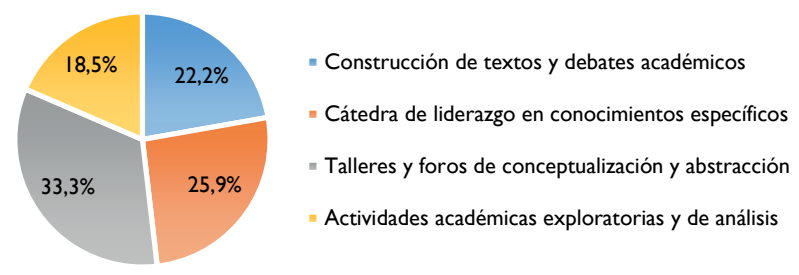

Figura 2. Relaciones entre las competencias investigativas y las prácticas laborales 
la seguridad humana y multidimensional, los modelos de enseñanza figuran como vehículos de transfiguración de la praxis profesional de los practicantes. A través de la investigación se fomenta primordialmente un ejercicio reflexivo y crítico que conllevará la adaptación a los nuevos roles que exige la seguridad. Por tanto, el método tradicional de enseñanza debe dar paso a modos de aprendizaje más flexibles y compatibles con las nuevas prácticas.

En comparación con las entrevistas semiestructuradas hechas a directivos, docentes y expertos en seguridad, se encuentran coincidencias con las expectativas y vivencias de los estudiantes sobre la necesidad de profundizar en contenidos interdisciplinares para la investigación, innovando las formas de aproximarse a la seguridad en la academia y en las diversas prácticas profesionales. A continuación, se muestran fragmentos relevantes de las entrevistas realizadas durante varios eventos académicos con la EPFAC, junto con el desarrollo del argumento alrededor de las capacidades y de los componentes para tener en cuenta en el replanteamiento de la MADGSI.

Las capacidades y las competencias como alternativas teóricas y pedagógicas para adaptarse a la seguridad contemporánea

Especialmente con los conceptos de seguridad humana y de seguridad multidimensional, la tarea de actualización de la política pública y de las doctrinas de seguridad es la profundización en el estudio de las relaciones cívicomilitares: ¿cuál es el papel de las agencias de seguridad en el desarrollo humano? Para responder esta incógnita debe acudirse a una reflexión que esté sustentada en el pragmatismo, en la acción autónoma basada en el conocimiento de sí mismo y de la situación actual.

Amartya Sen expone su entendimiento de la acción racional desde la centralidad de la libertad. El autor explica que la libertad para actuar, le otorga a la persona valor para obtener los resultados esperados e iniciativa para actuar efectivamente, para sí y para los otros; la libertad le da a la persona capacidad de agencia para participar de actividades sociales de diversas índoles (Sen, 1999). De hecho, la teoría de Sen se va a concentrar en la explotación de las capacidades para el desarrollo social e individual emplazando en su centro a la libertad de experimentar la vida más allá del simple objetivo de vivir; su teoría del desarrollo requiere que los individuos puedan decidir libremente bajo qué preceptos desean vivir y someterlos a la discusión pública (Sen, 1999).

Sen afirma que el objetivo primario del desarrollo es la expansión de la libertad; a su vez, esta libertad expresada en la capacidad de evitar los factores de su privación; e. g., hambruna, enfermedad, imposibilidad de participación política, censura, etc. (Sen, 1999). Es así como la capacidad se convierte en un elemento central para la acción de cualquier individuo o grupo, sin importar su condición socioeconómica, porque abre la posibilidad de defender su libertad de vivir según sus propios preceptos y aportar al desarrollo de la sociedad. No se trata de tener la posibilidad y la seguridad materiales como fines del desarrollo, sino del fortalecimiento institucional y normativo como medios para enriquecer la capacidad y la libertad de acción.

A partir de los supuestos de Sen sobre las capacidades, es posible contemplar que la educación posee un valor importante para potenciar la libertad debido a su injerencia de interseccionalidad con algunas dimensiones de la libertad. El caso directo, ilustrado por Sen, es el incremento del ingreso desde la inversión en la educación; las mejoras en la educación apuntan a un enriquecimiento de las capacidades de acción de los individuos y, por tanto, de su libertad. De esta forma, la educación en sus programas académicos debe evaluar qué principios y bajo cuáles competencias debe el estudiante desarrollar sus capacidades. Esto tiene unas implicaciones éticas importantes al incluir la responsabilidad del individuo con la aplicación de los conocimientos en la práctica, su impacto en la profesión y en los grupos sociales circundantes. Con respecto a la seguridad contemporánea, para superar los antiguos modelos de tratamiento de la seguridad nacional, debe suceder una renovación en la forma de enseñanza que permita la exploración de nuevas dinámicas y relaciones entre militares, policías y civiles (Sansó-Rubert, 20I3).

De acuerdo con Sen, la responsabilidad requiere de la libertad, puesto que su ejercicio depende de las condiciones contingentes de la experiencia personal y social (Sen, 1999). La posibilidad y libertad del individuo de involucrarse en los asuntos sociopolíticos se deben a los mismos contratos sociales, entonces la libertad del individuo no puede escapar a la responsabilidad con la sociedad. La responsabilidad social, entonces, es el encuentro del ejercicio de las libertades en interacción; dentro de esta responsabilidad se encuentra el deber por cuidar del otro y de las condiciones que permiten la explotación 
de las capacidades, e incluso cambiarlas (Sen, 1999). Conferir libertad con responsabilidad depende del aprendizaje del comportamiento ético y de la praxis adecuada a las contingencias emergentes; el militar y el policía deben conocer la integralidad de los conflictos y de los actores involucrados, al mismo tiempo que los límites jurídicos e institucionales de su acción y de su autoridad. En campos de acción tan heterodoxos respecto a la seguridad multidimensional, la educación de los encargados de esta debe extenderse más allá de la doctrina, hacia un aprendizaje guiado por la experiencia, la reflexión y la investigación.

Todo este proceso está sustentado en la capacidad reflexiva del individuo para evaluar sus propias condiciones y las interacciones con el entorno social, que será nutrida por la educación. Finalmente, Sen afirma que las capacidades humanas tienen una relevancia directa con el bienestar y la libertad, mientras que poseen un rol indirecto en el cambio social y en la producción económica (Sen, 1999). En consecuencia, esta reflexión lleva a preguntar: ¿el enfoque conceptual y pedagógico de la MADGSI les da libertad y capacidad a sus estudiantes para actuar?

Un buen reflejo de la educación en los escenarios de seguridad es proporcionado por las fuentes de la doctrina militar. Colombia, al ser un país con una altísima conflictividad histórica, ha formado a sus agencias de seguridad de manera innovadora, estratégica y operativamente. En buena medida, las fuerzas militares se han entrenado para la guerra irregular de guerrillas enfrentando los desafíos que cada contingencia histórica les imponía. Sin embargo, ahora el foco de la acción militar se está redireccionando con el surgimiento de otras amenazas de actual interés prioritario, como los crímenes cibernéticos, la minería ilegal, el tráfico de armas y de personas, el lavado de activos, el terrorismo, las catástrofes ambientales $y$, por supuesto, el narcotráfico.

Este cambio obliga a una transformación de la doctrina y de los métodos de enseñanza; el entrenamiento ha de diversificarse y a los militares especialmente deben impartírseles otras formas de relacionamiento y de acción frente a estas prioridades cambiantes de la seguridad, implicando una transformación en el pensamiento y en el conocimiento. En términos de Sen, los militares deben adaptar y desarrollar sus capacidades para ejercer sus nuevas funciones efectivamente. Si se entiende que la doctrina es, como componente de una estrategia general de seguridad, una serie de "prescripciones... [que especifican] cómo las fuerzas militares deberían ser estructuradas y empleadas para responder a amenazas y oportunidades reconocidas" (Posen, 1984, p. 13, traducción propia), su composición va a depender del relacionamiento con las concepciones básicas de la seguridad, especialmente con las experiencias más directas y cercanas.

Barry Posen descompone las diferentes doctrinas militares en tres clases: la doctrina ofensiva, cuyo objetivo se centra en el desarme o destrucción del otro; la doctrina defensiva, la cual persigue detener al otro de la consecución de su objetivo; y la doctrina disuasiva, una estrategia de castigo al otro (Posen, 1984). Para el caso colombiano, la doctrina de seguridad estuvo históricamente oscilando entre la ofensiva y la defensiva, debido a su concentración en los enemigos internos, guerrillas y organizaciones criminales. Sin embargo, dentro de las categorías de Posen, solo se encuentran respuestas a los desafíos clásicos de la seguridad nacional, y no hay una opción clara de una doctrina para la seguridad humana o multidimensional. De esta forma, se muestran insuficientes las antiguas concepciones de seguridad como las doctrinas basadas en ellas y sus métodos de enseñanza.

El desarrollo de una nueva doctrina acorde con la seguridad contemporánea, requiere de pensarse cuáles son las capacidades de las agencias de seguridad y cuáles son necesarias desarrollar. Cuando se trata de ciberseguridad, de desastres naturales o de minería ilegal, las acciones requeridas son diferentes a las emprendidas frente a un combate frontal contra otro ejército; las nuevas operaciones requieren de profesionalización adyacente al entrenamiento militar de acuerdo con las necesidades que propone cada amenaza y riesgo (Sansó-Rubert, 20I3). De allí se justifica la renovación del currículo de la MADGSI; de esta manera lo expresó un experto en seguridad durante el Foro Coladca, celebrado en Cali:

Los valores sociales, en general, y el respeto por el ser humano son esenciales. La transferencia de conocimiento sin ajustes a las necesidades particulares de las comunidades da como resultado una degradación de las estructuras sociales que ocasionan el surgimiento de múltiples factores de riesgo. Por esta razón, es fundamental el desarrollo del profesionalismo y de la ética en el gremio. Respecto al impacto del conocimiento en la seguridad, es necesario volver a lo fundamental en la construcción desde el ser, evaluarse desde qué currículo estamos trabajando en la construcción de seres íntegros. Una comunidad construida desde los 
valores la comunidad, en vez de ser reactiva, se convierte en proactiva, cuidar a los niños y a los adultos nos hace mejores sociedades.

De esta reflexión debe remitirse a un debate fundamental para esta discusión: la relación teoría-praxis. Se supone inmediata la aplicación del conocimiento en un ambiente que le demanda respuesta al actor, allí se hace explícito el valor del aprendizaje. Desde que el actor logra hacer su oficio de manera distinta y más eficiente, el conocimiento adquirido resalta su importancia. Sin embargo, lo que se busca en la educación posgradual es la profundización y especialización de un conocimiento previo; en la MADGSI se preconcibe un conocimiento básico sobre seguridad y temas afines a lo militar o policial, así se conforma un currículo con los contenidos que un profesional especializado en dirección y gestión de la seguridad debe manejar para mejorar sus prácticas laborales. De esta manera, se justifica la entrada de un enfoque de componentes en la estructuración curricular, definidos por Escrich, Lozano y García de la siguiente manera:

[...] una serie de conocimientos, habilidades, actitudes y aptitudes (que incluyan valores) que permitan desempeñar tareas no solo en un contexto dado, sino que [también] sean trasladables a otros, similares o no. Las competencias han de dotar a los titulados superiores de una serie de capacidades, de herramientas, que les permitan enfrentar esas demandas complejas, así como crear otras nuevas que les permitan tener un papel transformador en el entorno. Es decir, que los titulados sean verdaderos ciudadanos activos, participantes del cambio que afecta a sus propias vidas y a las del entorno en el que van a desarrollar sus actividades profesionales (Escrich, Lozano \& García, 2015, pp. 4-5).

En consecuencia, a partir de la inclusión de los componentes al proceso de reestructuración de la MADGSI, el estudiante podrá adaptarse efectivamente a nuevos desafíos profesionales. Las competencias guían el aprendizaje hacia el desarrollo de habilidades de uso práctico para actuar diferente y poder efectuar cambios, gestionar programas de seguridad y de eliminación de riesgos. En síntesis, se trata de fomentar una visión estratégica para coordinar acciones complejas para atacar estructuralmente las fuentes de riesgo. De esta forma, lo expresa el experto en seguridad:

Un riesgo puede ser eliminado, esto a partir de conocer el alcance en la gestión del riesgo; hay que identificar las fuentes antes que los riesgos, lo que inmediatamente permite la integración de la gestión de riesgos. La fuente de riesgo es la que se aprovecha de la vulnerabilidad, entonces hay que controlar la fuente generadora. La gestión del riesgo debe estar adaptada al negocio, esto implica que debe haber un conocimiento entre el contexto interno y externo. La gestión del riesgo puede concebir la visión de aumentar el riesgo si se persiguen oportunidades. Se puede y se deben integrar todos los sistemas de gestión.

En última, los componentes presentes en la formulación del currículo y de las metodologías de enseñanza deben incluir primordialmente el crecimiento de las capacidades de acción de los estudiantes posgraduales. La conjugación de capacidades y componentes conllevaría la formación de graduados autónomos, críticos y reflexivos debido a que el concepto de capacidad

permite que la competencia no se convierta en un simple medio para alcanzar un fin... sino que pueda considerarse un fin en sí mismo (estar dotado de determinadas habilidades o contar con las herramientas necesarias para desarrollar un trabajo de forma productiva) (Escrich, Lozano \& García, 2015, p. 20).

Consecuentemente, los componentes que deben regir el programa de MADGSI deben estar establecidos a partir de la reflexión sobre las necesidades de las seguridades multidimensional, humana e integral. Las acciones subsiguientes requerirán del análisis acerca de las necesidades metodológicas, tecnológicas y pedagógicas que sirvan para el desarrollo del programa, desde las actualizaciones en los componentes basados en capacidades. Así lo expresa una docente de la EPFAC:

Lo primero es fortalecer el tema de participación en semilleros, donde el desarrollo de investigaciones reales los estudiantes participan en la búsqueda de información, elaboración de fichas bibliográficas, etc., con la intención de que el estudiante aprenda a investigar en lo práctico. Los ritmos de otros compromisos de la escuela pueden afectar, pero se trata de regularizar el proyecto, por eso el estudiante debe ser inquieto, indagar, aprender a ser sistemático de la información manejando software y herramientas de búsqueda. También debe intensificarse la participación en eventos de divulgación científica, que conozcan cómo se hace la ponencia, la evaluación a un artículo $y$, en general, eventos de redes y desarrollo de trabajos y productos de investigaciones. Es importante tener en cuenta las transformaciones de las fuerzas en términos de formación académica. 


\section{Conclusión}

Los resultados de la encuesta y de las entrevistas apuntan a que los nuevos desarrollos del programa de MADGSI, identificado por estudiantes, docentes y expertos, deben enfocarse en una formación que priorice a la investigación como práctica para resolver problemas que imponen los riesgos y las amenazas a la seguridad de hoy. En especial, el currículo debe direccionar, desde los componentes, las capacidades de los estudiantes a identificar el riesgo, planificar una estrategia integral, disponer de los recursos necesarios y activar los protocolos y mecanismos funcionales para contrarrestar las eventualidades ya previstas. Además, para la toma de decisiones reactiva, el estudiante mediante la investigación adquiere experiencia en los razonamientos prácticos, métodos innovadores y conceptos útiles que sustentan las capacidades de decidir libre y racionalmente. Continúa la docente de la EPFAC:

Hay que cumplir los estándares con educación, por tanto, los docentes mejor preparados deberían estar orientados hacia estos. No hay comunicación y debe haber integración entre títulos y coherencia de investigación. Si el estudiante no tiene nivel, entonces hay que desarrollar el tema desde pregrado y continuar el proceso en el posgrado; en temarios concretos una formación integral en investigación. De la misma manera, impactar la progresión de los estudios de las personas tanto en la carrera militar como en la vida civil.

En este apartado se menciona un punto de inflexión para la educación superior y posgradual, que lleva al cuestionamiento de la calidad de la educación universitaria general: la continuidad entre pregrado y posgrado. Allí se encuentra un reiterado argumento que destaca una falencia generalizada de los profesionales que buscan especializarse, en tanto carecen de conocimiento y práctica de investigación. No obstante, debería estar contemplado dentro de la educación por componentes, no solo el fortalecimiento metodológico y teórico de los estudiantes, sino también una más fuerte aproximación con la sociedad para enriquecer la experiencia investigativa con la realidad social, en todas sus diversas dimensiones. Para este caso, esta afirmación implica que la educación militar y policial debe ser más aguda en el desarrollo de capacidades mediante el fortalecimiento de las competencias investigativas; de esta forma se lograría aumentar el conocimiento sobre el campo de trabajo y mejorar las prácticas de seguridad ambiental, cibernética o de frontera.
Si la práctica investigativa dota de nuevas capacidades de acción al estudiante de la MADGSI, es fundamental señalar que sus componentes deben incluir: el análisis y abstracción de información, la actualización teórica en gestión del riesgo, el conocimiento suficiente de la normatividad y sus aplicaciones, la afinación de la capacidad de decisión y reacción, el aumento de la capacidad de identificación de problemas y riesgos, la precisión de la aplicación de estándares y normas, y el fomento de la actualización permanente en los diversos temas que incluyen la seguridad integral. Estas competencias tendrían un efecto importante para la competitividad por área de trabajo dentro de la seguridad, dado que le otorgan al profesional un valor agregado cuando se destaca su visión integral de la seguridad y su pragmatismo en temas gerenciales y de planeación.

Es claro que no puede obviarse el origen militar de la MADGSI, como programa de la EPFAC. Por tanto, las transformaciones curriculares y pedagógicas que sean posibles de efectuar dentro del programa deben comprender y corresponder a la complementariedad del enfoque de competencias con el entrenamiento y la doctrina militar: por una parte, la disciplina y el compromiso como principios éticos de responsabilidad del profesional con las dinámicas complejas de la seguridad humana; por otra, el respeto por la institucionalidad y la garantía del cumplimiento de la ley y de la protección de los derechos humanos como límites normativos y éticos de la formación y práctica profesional. La ética del servicio incorporada dentro de las nuevas prácticas es esencial para la conducción exitosa de programas, operaciones, protocolos e incluso, organizaciones de seguridad. De esta forma, también es necesario hacer revisión de un debate sobre la ética entre las nuevas seguridades y las innovaciones procedimentales de las fuerzas militares y policiales. Es imperativo resaltar que las transformaciones estructurales requeridas dentro de la doctrina no cambiarán solo por voluntad política, deben estar acompañadas de cambios culturales y normativos que ajusten efectivamente la funcionalidad y las capacidades de las agencias de seguridad a las amenazas y riesgos actuales.

Además, en lo relativo a las relaciones cívico-militares, el desarrollo de capacidades a través de los componentes previamente mencionados resulta ser de vital importancia por la exploración imperativa del profesional con diversas prácticas de investigación, que a su vez lo llevarán necesariamente a concebir nuevos relacionamientos e interacciones con la sociedad civil. Los nuevos desafíos a la seguridad demandan el acercamiento de las fuerzas militares a los 
grupos sociales, sean organizados como empresa privada u organizaciones no gubernamentales; esto con el objetivo de disminuir los riesgos internos a través del aumento de la confianza pública de las instituciones de seguridad y con el conocimiento cercano del funcionamiento interactivo y simbólico de la sociedad. Para la consecución de este último objetivo, la investigación debe posicionarse como una herramienta fundamental en el desarrollo de estas relaciones cívico-militares, a modo de ampliar la comunicación entre las fuerzas militares y policivas con la sociedad civil, buscando las fuentes de la seguridad y de las amenazas y riesgos al interior de las interacciones sociales, económicas, políticas, culturales y tecnológicas. De esta manera, la profesionalización de los agentes de seguridad se asegura como integral e innovadora, con capacidad de gestión a gran escala de las necesidades y expectativas de la sociedad en los aspectos relativos a la seguridad.

Se recomienda, no solo la aplicación de estas consideraciones de reforma en el programa académico de la MADGSI, sino también en la formulación de política pública para la seguridad; la ampliación del entendimiento del panorama completo permite el diseño innovador y creativo de las soluciones en programas y planes basados en la realidad y en la evidencia, lugar donde la investigación en campo desempeña un papel fundamental. La política pública podría servirse, por una parte, del enfoque de capacidades de Sen para mejorar los estándares de vida y de libertad de los ciudadanos $y$, por otra, de los componentes como referentes analíticos del estado de las situaciones para fomentar el mejoramiento de las habilidades $y$ capacidades de acción de los sujetos. En general, la fórmula capacidad-componente permite una planeación reflexiva de lo que necesitan los sujetos y de lo que serían capaces de ser y hacer mediante una formación integral que les dé la posibilidad de actuar libremente y de manera diferente frente a contextos nuevos y desafiantes. En materia de seguridad, se pretende enlazar efectivamente la teoría con la praxis, de modo que se fortalezcan las capacidades, a modo de libertad responsable, en el trabajo.

En última instancia, es importante mencionar la relevancia de las relaciones interinstitucionales en el desarrollo de un proyecto educativo basado en el enfoque capacidadcomponente, dado que la cooperación entre diferentes clases de instituciones puede facilitar recursos y experiencias a los estudiantes y profesionales para ampliar sus aprendizajes y conocimientos prácticos. La extensión teórica y metodológica de la MADGSI debe también contemplar la triada Estado-universidad-empresa (Sábato \& Botana, 1975) para enriquecer los horizontes teórico-prácticos que afrontan los estudiantes en sus labores de seguridad.

\section{Reconocimientos}

Este artículo es un producto de investigación derivado del proyecto Impacto de las políticas de seguridad integral en el desarrollo y gestión del componente de investigación del currículo MADGSI. Convocatoria Colciencias 995-2017 INVITACIÓNA PRESENTAR PROPUESTAS PARA LA EJECUCIÓN DE PROYECTOS DE I+D+i - FAC.

\section{Referencias}

Aparicio, J. (2019). De la seguridad nacional a la seguridad ciudadana: el papel de la Policía Nacional. Bogotá: Policía Nacional de Colombia.

Beck, U. (2008). ¿Qué es la globalización?: falacias del globalismo, respuestas a la globalización. Barcelona: Paidós.

Booth, K. (2007). Theory of world security. Cambridge: Cambridge University Press.

Denzin, N. (1978). The research act:A theoretical introduction to sociological methods. New York: McGraw-Hill.

EPFAC. (20I5). Documento maestro para la solicitud del registro calificado del programa de Maestría en Dirección y Gestión de la Seguridad Integral.

Escrich,T., Lozano, F., \& García,A. (25 de junio de 2015). Competencia vs. capacidades: ienfoques complementarios o excluyentes? XXIV Jornadas de Economía de la Educación. Madrid.

Johnston, R. (2008). Survey methodology. En Box-Steffensmeier, J., Brady, H., \& Collier, D. (Eds.). The Oxford Handbook of Political Methodology (Pp. 385-403). Oxford: Oxford University Press.

Morgenthau, H. (1948). Politics among nations: The struggle for power and peace. New York:Alfred A. Knopf.

OEA. (2003). Declaración sobre seguridad en las Américas.

Peoples, C., \& Vaughan-Williams, N. (2010). Critical security studies:An introduction. London: Routledge.

Posen, B. (1984). The sources of military doctrine: France, Britain and Germany between the world wars. New York: Cornell University Press.

Sábato, J., \& Botana, N. (1975). El pensamiento latinoamericano en la problemática ciencia-tecnología-desarrollo-dependencia. Buenos Aires: Paidós.

Sansó-Rubert, D. (20I3). La seguridad ciudadana y las Fuerzas Armadas: ¿despropósito o último recurso frente a la delincuencia organizada? Revista Criminalidad, 55(2), I I9-133.

Sen, A. (1999). Development as freedom. New York: Alfred A. Knopf.

Thelier, T. (2010). Societal security. En Dunn, M., \& Mauer, V. (Eds.). The Routledge Handbook of Security Studies (pp. 105I I5). New York: Routledge. 